\title{
Review of wideband MIMO channel measurement and modeling for IMT-Advanced systems
}

\author{
ZHANG JianHua \\ Key Laboratory of Universal Wireless Communications of Ministry of Education, Beijing University of Posts and Telecommunications, Beijing \\ 100876, China
}

Received December 18, 2011; accepted February 20, 2012

\begin{abstract}
In October 2005, the next generation mobile communication system was officially named as the International Mobile Telecommunications-Advanced (IMT-Advanced) by the ITU Radiocommunication Sector (ITU-R). Wideband multiple input and multiple output (MIMO) channel models in multiple propagation environments are fundamental for IMT-Advanced systems and such research has been initiated since 2003. The research challenges come from the fact that the wider bandwidth (20-100 MHz) and the advanced multiple antenna technology have been proposed for the IMT-Advanced system, thus leading to higher sampling rates and multiple spatial propagation channels. In this review, four aspects of wideband MIMO channel measurement and modeling are discussed: (1) radio channel measurement procedure and equipment; (2) large scale fading models; (3) small scale fading models; and (4) MIMO channel models. In particular, the large scale fading affected by the carrier frequency is investigated for urban macrocells and it shows that the higher carrier frequency results in greater loss for non-line-of-sight (NLoS) conditions in the cities of China, for which the frequency dependent factor is 32.1. Moreover, the dense and the obviously higher buildings also lead to a larger angle spread (AS) of both the angle of arrival and angle of departure in urban macrocell scenarios. The results indicate that there is the potential to explore the MIMO technique for an IMT-Advanced system with larger ASs, which would lead to the high system capacity. The progress on MIMO models is described and some methods for simplifying geometry-based stochastic channel models (GBSM) are proposed. Finally future research topics on channel measurement and modeling are identified.
\end{abstract}

next generation communication systems, IMT-Advanced, channel, wideband, MIMO, measurement, modeling

Citation: Zhang J H. Review of wideband MIMO channel measurement and modeling for IMT-Advanced systems. Chin Sci Bull, 2012, 57: 2387-2400, doi: $10.1007 /$ s11434-012-5203-2

With his experiment to transmit and receive radio waves in 1887 experiment, Hertz started a new era for wireless communication. Over the past several decades, wireless mobile communication system has experienced rapid development from the first generation analogue system in 1980 s to the third generation digital system around 2000 [1]. In October 2005, the ITU Radiocommunication Sector (ITU-R) officially named the fourth generation mobile communication system as the International Mobile Telecommunications-Advanced (IMT-Advanced) systems [2]. Its overall objectives [3] are to realize $100 \mathrm{Mb} / \mathrm{s}$ for low-

email: jhzhang@bupt.edu.cn speed data rates, and $1 \mathrm{~Gb} / \mathrm{s}$ for high-speed data rates. To achieve such high data rates, the IMT-Advanced system bandwidth needed to be expanded to $100 \mathrm{MHz}$ and wireless access technologies are expected to achieve high spectral efficiency of the order of $10 \mathrm{bit} \mathrm{s}^{-1} \mathrm{~Hz}^{-1}$.

For a wireless communication system, the channel is the transmission medium between the transmitter (TX) and receiver (RX). Channel measurement involves collecting experimental data on the propagation characteristics of specified environments, also known as channel sounding from the point of probing the environment by sending out a known signal. Based on such data, a mathematical model can then be constructed in a form suitable for evaluating the 
performance of a proposed system. The propagation characteristics of the radio channel determine the performance limit of a wireless communication system [4].

Thus, channel measurement and the modeling of propagation characteristics are crucial in the research of new technologies, the design of new systems, the evaluation of standardization proposals, and the actual deployment of wireless communication systems. For example, with the assumption of independent and identical distributed (i.i.d.) Rayleigh fading channels, Winters [5], Foschini and Gans [6] and Telatar [7] discovered that multiple-input multiple-output (MIMO) technology provides remarkable spectral efficiency when the channel exhibits rich scattering. Their pioneering work initiated enormous interest in MIMO technology and created a new era of mobile communications.

In the 1940s, Rice started the research on stochastic channel modeling theory [8]. In the 1950s, Lincoln Laboratory of the Massachusetts Institute of Technology (MIT) proposed a two-dimensional random process in both time and frequency to describe the wireless mobile communication channel. Since the 1970s, researchers in Europe and North America initiated the development of a time-frequency domain sounder. They then carried out a large amount of radio channel measurement under various complicated environments such as urban, suburban and indoor scenarios. Based on such empirical channel data, a series of channel models were proposed for a wireless mobile communication system. Their achievements are included in the European Cooperation in Science and Technology 207 (COST 207) and ITU-R M.1225 [9,10], which are widely used in the development and evaluation of the second and the third generation mobile communication systems.

With the requirements of the IMT-Advanced system for high spectral efficiency and higher data rate services, more spectrum (450 MHz-5 GHz), wider bandwidth (20-100 $\mathrm{MHz}$ ) and advanced MIMO technology have been proposed. Thus, the wideband MIMO channel model covering from $450 \mathrm{MHz}$ to $5 \mathrm{GHz}$ band is mandatory [11,12]. In order to solve such challenges to channel models, Europe launched the Wireless World Initiative New Radio (WINNER) [13] project as early as 2003. Research institutions and companies in Japan, South Korea, and United States etc. also carried out corresponding research [14,15].

It is well-known that channel propagation characteristics are determined by geographical profile and population distribution, etc., especially in an urban environment. Because there are considerable differences between China and other countries in population density, vegetation and building distribution, a channel model that reflects the Chinese propagation environment characteristics is required for the design, evaluation and application of IMT-Advanced systems in China. As a consequence, since 2004 our team has been conducting channel measurement and modeling for wideband MIMO systems in typical deployment scenarios in China [16-19]. Our contribution to wideband MIMO channel measurement and modeling includes:

(1) Considering the fact that high-rate data services are found mainly in crowded shopping malls, conference venues, etc., the propagation characteristics in such indoor hotspot scenarios are thoroughly investigated. The results have been adopted by ITU-R M.2135 as one of four mandatory evaluation scenarios of the IMT-Advanced system [20-22].

(2) Considering the fact that the high population in China leads to high density and average heights for buildings, the propagation characteristics in a typical urban macrocell, urban microcell and rural macrocell in China are analyzed and our results are clearly different from those of WINNER.

(3) Based on the measured data, we found low correlation between the $K$-factor, angle of arrival (AoA) and angle of departure (AoD), etc., and five methods for simplifying geometry-based stochastic channel models (GBSM) are proposed, and a cluster deleting scheme that would reduce the simulation time by as much as $40 \%$ when a $25 \mathrm{~dB}$ clipping threshold is adopted for the "Indoor LoS" case.

This review presents the progress of wideband MIMO channel measurement and modeling in recent years, especially for IMT-Advanced systems. Empirical models are constructed with measured data and compared with those of WINNER. The latest results in channel model simplification are noted and finally some future research topics on channel measurement and modeling are identified.

\section{Procedure for radio channel measurement and modeling}

Research on the characterization of radio propagation is based on field measurements. First we need to collect data from various scenarios with different frequencies and antenna configurations using a channel sounder. The necessary channel parameters are then extracted by advanced data processing algorithms. The stochastic property of channel fading is mathematically described and, finally, a radio channel is reconstructed in a form suitable for evaluating the performance of a proposed system.

The channel sounder is the instrument that determines the resolution of the observed channel in the time-frequencyspace domains. Research and development on channel sounders began in the 1970s [23]. Professor Cox [24] from Stanford University used a spread spectrum sliding correlator to develop a sounder. Subsequently, a narrowband sounder working at $800 \mathrm{MHz}$ for second generation communication systems was developed with a direct radio frequency (RF) pulse technique. The high requirements of the IMT-Advanced system in bandwidth and spatial properties pose a great challenge for a channel sounder. An advanced channel sounder is indispensable [25], and must have the capability to synchronize accurately between transmitter 
and receiver, wider bandwidth (up to $100 \mathrm{MHz}$ ), high storage capacity and high speed data collection etc. In recent years, PropSound CS developed by Elektrobit of Finland and RUSK developed by MEDAV of Germany have been viewed as two of the most representative MIMO channel measurement items of equipment in the world. Both of them are widely used in well-known projects such as WINNER [13].

Figure 1 shows the working principles of PropSound CS, and working mode based on a Time Division Multiplexing MIMO (TDM-MIMO). The working procedure is as follows:

(1) Transmitter: generates modulated specific pseudo-noise (PN) codes, which will be transmitted through the selected transmitting antennas in turn. The antenna switching is controlled by a high-speed switch unit.

(2) Receiver: the antennas receive the signal in turn. After down-conversion, the baseband signal will be coherently demodulated to acquire the channel impulse response (CIR). A real-time result display helps to determine the validity of the raw measurement data, and this will eventually be stored in a prescribed format for post-processing.

Due to the above demanding requirements, the channel sounder is usually very expensive and thus only about ten sets are owned by operators and research institutes in the entire world, for example by the University of Oulu in Finland [26], University of Ilmenau in Germany [27], Univer- sity of Surrey in United Kingdom [28], China Mobile Research Institute in China, Stanford University in United States [29] and DoCoMo in Japan. In 2004, we used a MIMO-OFDM hardware platform with a bandwidth of 20 $\mathrm{MHz}$ as the channel sounder [30]. However this system adopted a Code Division Multiple Access MIMO (CDMAMIMO), where the interference between multiple pseudo-random (PN) sequences may exist. Due to the non-ideal cross-correlation characteristics of PN codes, performance degradation occurs at low signal to noise ratios (SNRs). Taking these factors into consideration, in 2005 we hired a PropSound CS to conduct channel measurements in indoor hotspots and outdoor scenarios. In 2007, China Mobile Research Institute purchased a PropSound CS for the purpose of providing empirical channel data and models to mobile operators in China for the design and optimization of their mobile networks.

By using a channel sounder, CIRs can be acquired, which describe the channel propagation properties and provide the data basis for extracting other statistical parameters. The first step in data analysis is to calculate CIRs from raw measurement data. Subsequently, other statistical parameters and models can be acquired. Figure 2 lists all the channel characteristic parameters, which are important for a wireless system. These parameters and models are usually categorized into large scale and small scale fading. The former can be calculated directly from the CIRs. For small

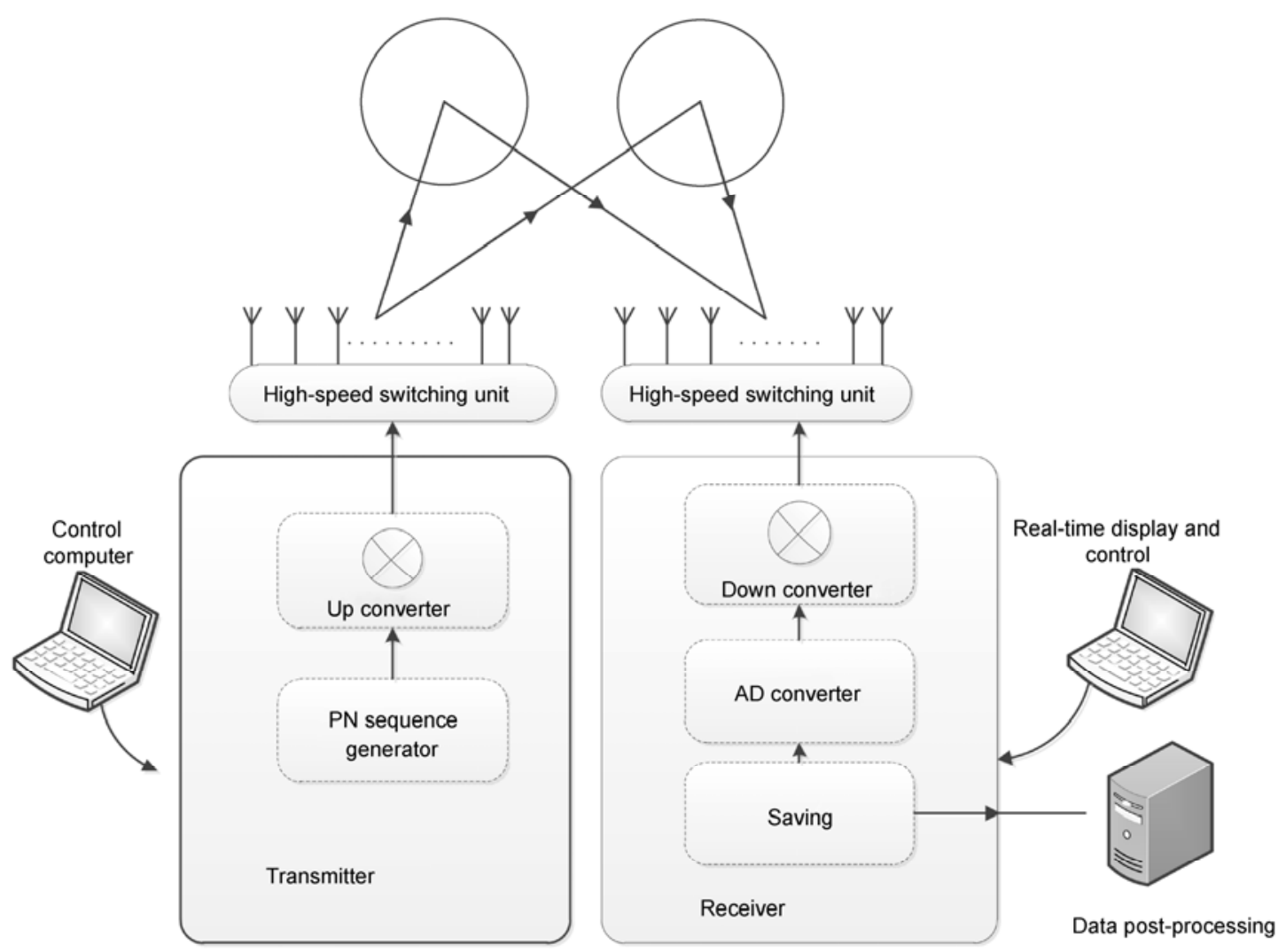

Figure 1 Working principles of the channel sounder: PropSound. 


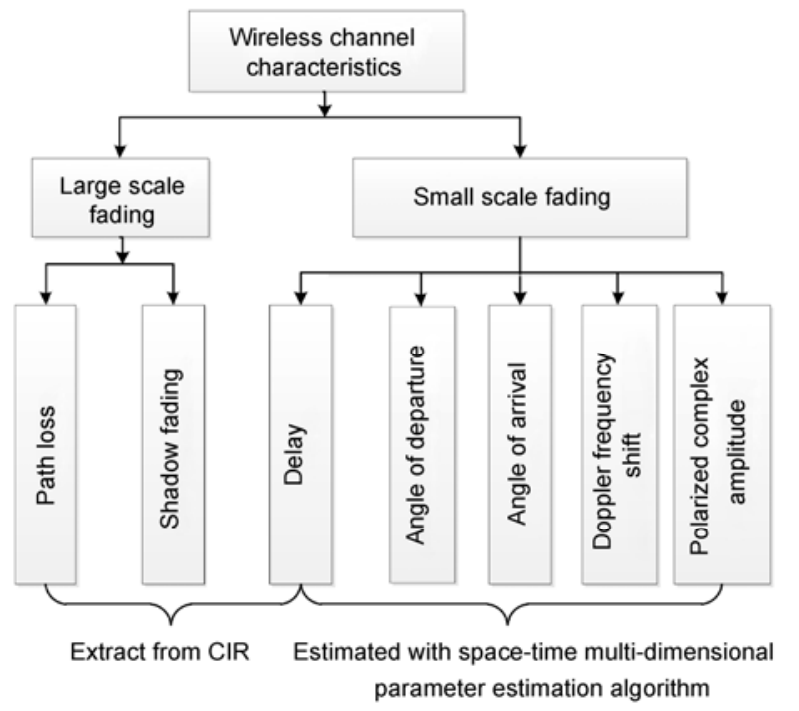

Figure 2 Channel property parameters.

scale fading, the channel parameters, including delay spread, AoD and AoA, Doppler frequency shift and complex magnitude of polarization, are acquired by first using a multi-dimensional joint parameter estimation algorithm. Empirical models can then be constructed on the basis of these parameters. Large scale fading mainly has an impact on the average receiving power whereas delay spread and angular spread determine the extent of frequency and spatial selective fading. Doppler frequency shift and polarization are also included in the measurement based channel model. The process after acquiring the CIRs is described in Sections 2 and 3 .

\section{Large scale fading channel models}

The fluctuation of received power averaged for a local area of about $10-40 \lambda$ (where $\lambda$ is the wave length), is known as large scale fading. Large scale fading includes shadow fading caused by large obstacles and path loss caused by the fact that received power decreases as the distance between RX and TX increases. Large scale models are essential for analyzing the coverage, planning and optimization of a wireless system.

So far, almost all the large scale fading models are based on the single-slope log-distance model, i.e.,

$$
P L(d)=P L_{0}+10 n \cdot \log _{10}(d)+X_{\sigma},
$$

where $P L_{0}$ is the intercept value of path loss, $n$ is the path loss exponent, and $X_{\sigma}$ is a log-normal distribution random variable with standard deviation $\sigma$ (in $\mathrm{dB}$ ), which indicates the variability of the received power at the same distance $d$ between RX and TX.

The Okumura-Hata model for the macrocell is the most widely used model for the second generation communica- tion systems at 900 and $1800 \mathrm{MHz}$. This was proposed by Okumura et al. [31] based on considerable measurements in Japan and further improved by Hata [32]. In microcells and macrocells with small coverage, the COST 231-WalfishIkegami model is generally used. This model is based on the research of Walfisch and Bertoni [33] and Ikegami et al. [34] etc., which was proposed in the COST 231 [35] project framework. Recently, the WINNER project conducted measurements in various scenarios from 2 to $5 \mathrm{GHz}$ and developed corresponding path loss models.

Since 2005, we have conducted numerous measurements in typical scenarios, including indoor hotspots, outdoor microcells and macrocells etc. The large scale fading models were extracted and compared with European results. We paid particular attention to the hotspot scenarios because the higher peak data rate services usually occur in these scenarios and more than $50 \%$ of the calls are initiated from indoor localities. Consequently the indoor hotspot is one of the typical and important scenarios for IMT-Advanced systems which was proposed by us to ITU-R and accepted as one of four mandatory evaluation scenarios for these systems.

In our measurements, we chose a teaching building with a wide corridor and several large multi-media classrooms as indoor hotspot scenarios. Figure 3 shows the layout of the first floor, including fixed measurement points and mobile routes in the indoor section and outdoor-indoor section. All the points in Grid A are line of sight (LoS) propagation cases. Some of the points in Grid B are LoS propagation cases and the rest are obstructed-LoS (OLoS) propagation cases. All the points in Grid C are Non-LoS (NLoS) propagation cases, and all the points in Grid D are outdoor-indoor propagation cases. Paths $\mathrm{E}$ and $\mathrm{F}$ are the mobile measurement routes. The external wall and internal walls are made of reinforced cast concrete, whereas the ground inside the building is paved with marble. In addition the door of the entrance is made of transparent glass and all other doors are made of wood. All the doors are kept closed during the measurements.

Fitting the measurement data to the large scale fading model for the LoS propagation case gives:

$$
P L(d)=39.2+16.9 \log _{10}(d) \quad \sigma=1.3 \mathrm{~dB},
$$

where the path loss exponent at 1.69 is smaller than 2 in free space because indoor LoS propagation is in a relatively enclosed area with reflection caused by roofs, floors, walls and other large objects, and scattering caused by numerous objects. The received power is the sum of the many arrival paths, which is naturally higher than that in free space. Many studies have demonstrated similar results. For example, a measurement was conducted at $5.3 \mathrm{GHz}$ [36] in a departure lounge scenario by the European WINNER project staff, and the path loss exponent was 1.3-1.5 for LoS propagation. Ref. [11] shows the exponent of path loss of 1.6 in a lecture hall and 1.42 in a stadium at $800 \mathrm{MHz}$ for LoS propagation. 


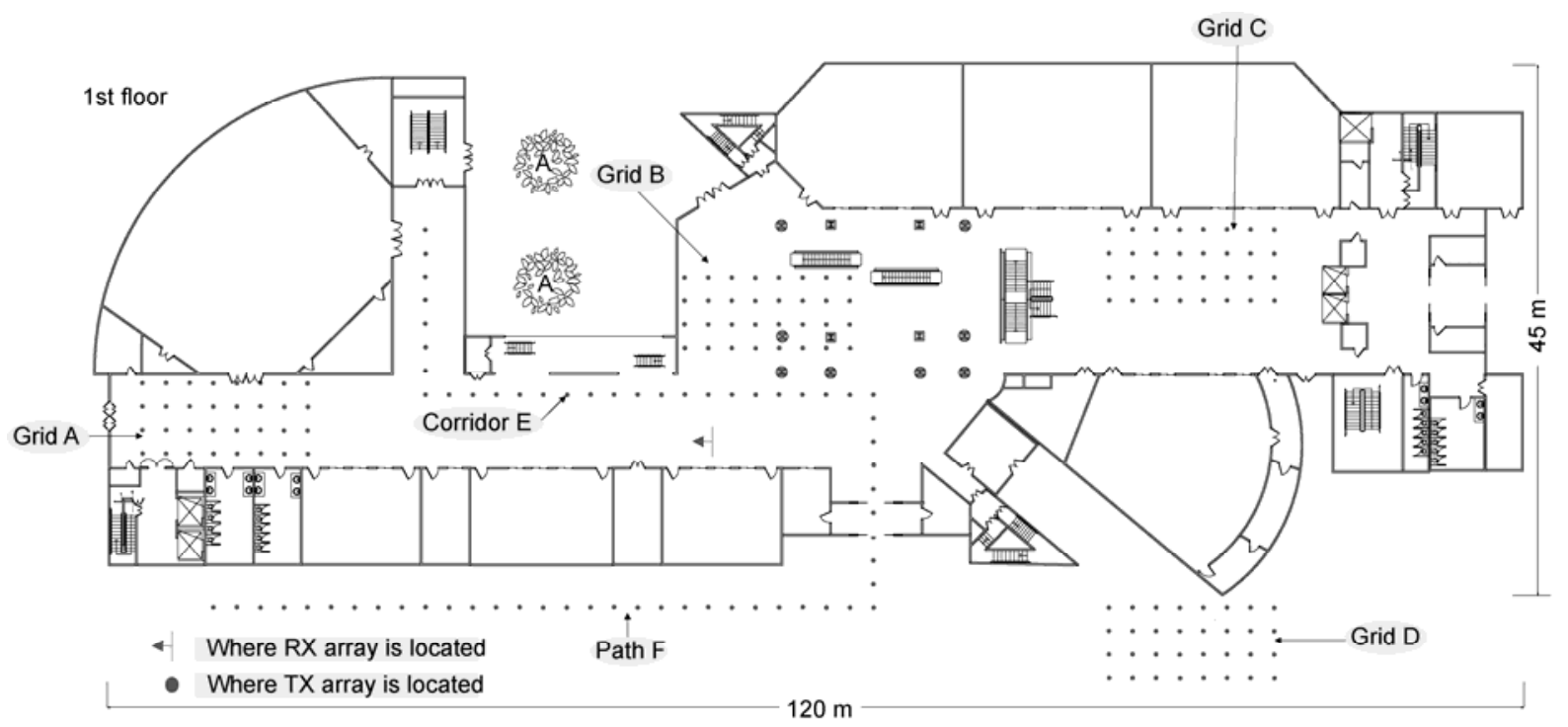

Figure 3 Planning of indoor hotspots and outdoor to indoor scenarios.

In the NLoS case, radio waves may be obstructed by walls and floors, so the received signal can be seriously attenuated. When $d=40 \mathrm{~m}$, the difference in the path loss between NLoS and LoS is $26.5 \mathrm{~dB}$. The model for the indoor hotspot scenario in NLoS is expressed as:

$$
P L(d)=25.5+43.3 \log _{10}(d) \quad \sigma=1.1 \mathrm{~dB},
$$

where the path loss exponent is 4.33 , which demonstrates the increasing trend of path loss with distance in NLoS case is more obvious than that in LoS case.

It is known that the frequency bands allocated for IMTAdvanced systems range from $450 \mathrm{MHz}$ to $5 \mathrm{GHz}$ and the propagation characteristics of the channel depend on the carrier frequency. To investigate the relationship between carrier frequency and large scale fading, a frequencydependent factor (FDF) $C$ is introduced and the large scale fading model is revised as:
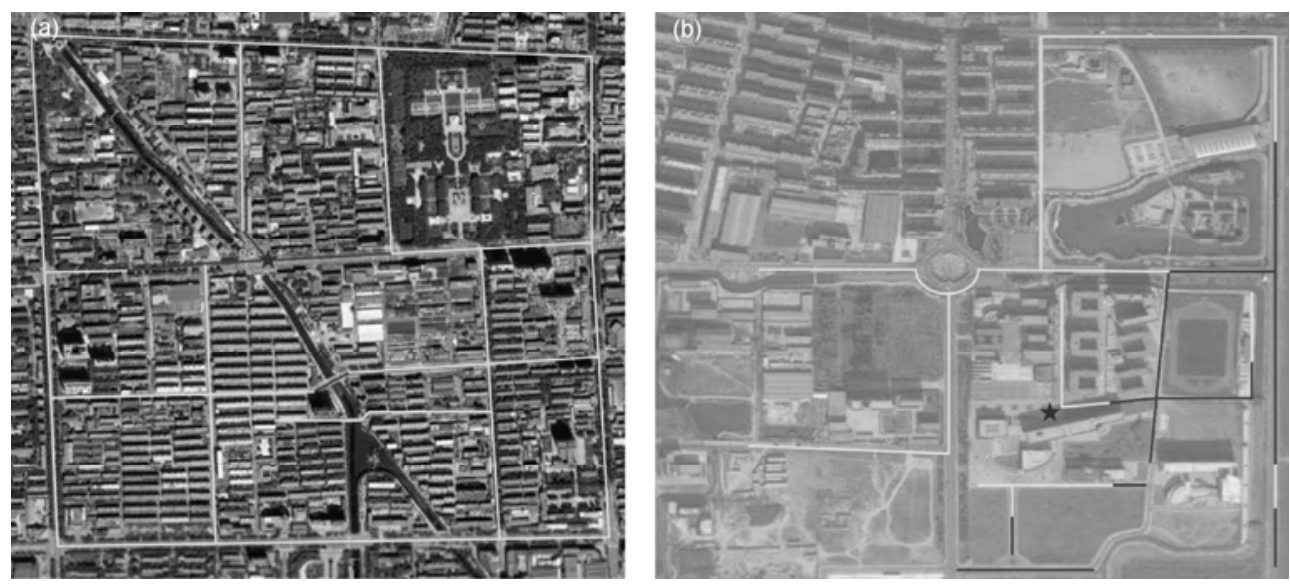

Figure 4 Roadmap in SJZ (a) and Roadmap in BJ (from Google map) (b). $\star$, BS location.

$$
\begin{aligned}
P L_{f_{0}}(d)= & P L_{0}+10 n \cdot \log _{10}(d)+X_{\sigma} \\
& +C \cdot \log _{10}\left(f_{c} / f_{0}\right),
\end{aligned}
$$

where $f_{0}$ is the reference carrier frequency and $f_{c}$ is the system frequency. For the free space model, $C=20$. Several measurement experiments were conducted in urban macrocell and microcell scenarios in Beijing (BJ) and Shi Jiazhuang (SJZ) (Figure 4) respectively. The measurement environment in BJ was a typical macrocell scenario where the average height of the buildings was lower than the transmitter. The receiver was set on the top of a van and driven around the measurement routes. For the urban microcell scenario in SJZ, the transmitter was lower than the average height of the surrounding buildings, and the density of the buildings was greater than that in an urban macrocell. The detailed system parameters are listed in Table 1. A threedimension omni-directional array (ODA), as in Figure 5(a),

\footnotetext{
Figure 4. Roadmap in SJZ (a) and Roadmap in BJ (from Google map) (b). $\star \star$, BS location.
} 
Table 1 Measurement parameters

\begin{tabular}{lcccc}
\hline Scenario & \multicolumn{2}{c}{ Microcell } & \multicolumn{2}{c}{ Macrocell } \\
\hline Carrier frequency & $2.35 \mathrm{GHz}$ & $580 \mathrm{MHz}$ & $2.35 \mathrm{GHz}$ & $4.9 \mathrm{GHz}$ \\
Code rate $(\mathrm{MHz})$ & 100 & 25 & 100 & 100 \\
Bandwidth $(\mathrm{MHz})$ & 200 & 50 & 200 & 200 \\
PN length & 1023 & 1023 & 1023 & 1023 \\
Transmitted power & 33 & 33 & 40 & 40 \\
Antenna in basestation & UPA & UPA & UPA/VDA & UPA/VDA \\
Antenna in UE & ODA & VDA & ODA & ODA \\
Sub-channels & $32 \times 8$ & SJZ: & BJ: $16 \times 8$ & BJ: $16 \times 8$ \\
Channel sampling rate & 160.2 & 21.2 & 436.4 & 614.8 \\
\hline
\end{tabular}
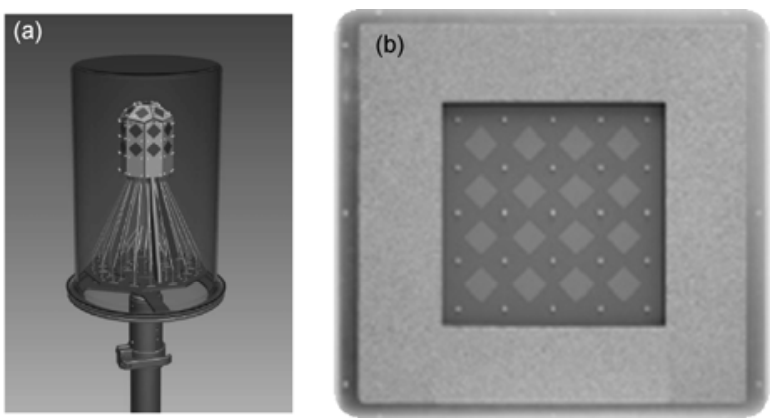

Figure 5 ODA antenna array (a) and UPA antenna array (b).

with 56 antennae was installed at the mobile terminals, while a uniform planner array (UPA), as in Figure 5(b), was used at the base station. The distance between the ODA and UPA antenna arrays is half a wavelength. In our measurements, the base station used a UPA with 8 antenna arrays, while the mobile terminals used an ODA with 32 or 16 elements antenna array. To compare the large scale fading in different carrier frequencies, we used a vertically-polarized dipole antenna (VDA) in the macrocell scenario in SJZ.

From the measured data, we found $C=19.8$ in the LoS case for an urban macrocell as shown in Figure 6(a), which is similar to free space. WINNER also gave an FDF of 20 from 2 to $6 \mathrm{GHz}$ within the break-point distance. However, in the NLoS case, $C=32.1$ as shown in Figure 6(b), which was much larger than the 23 given by WINNER, so the higher carrier frequency resulted in a greater loss for the NLoS case in the cities of China and any future system at a higher carrier frequency would require greater transmitting power to cover the same area.

\section{Small scale fading models}

In a small area (about $1 \lambda$ ), the received power fluctuates around a local mean value. Such fluctuation is called multipath fading (also known as small scale fading). It is essential to be familiar with the property of small scale fading for the design of and research into the transmission technology
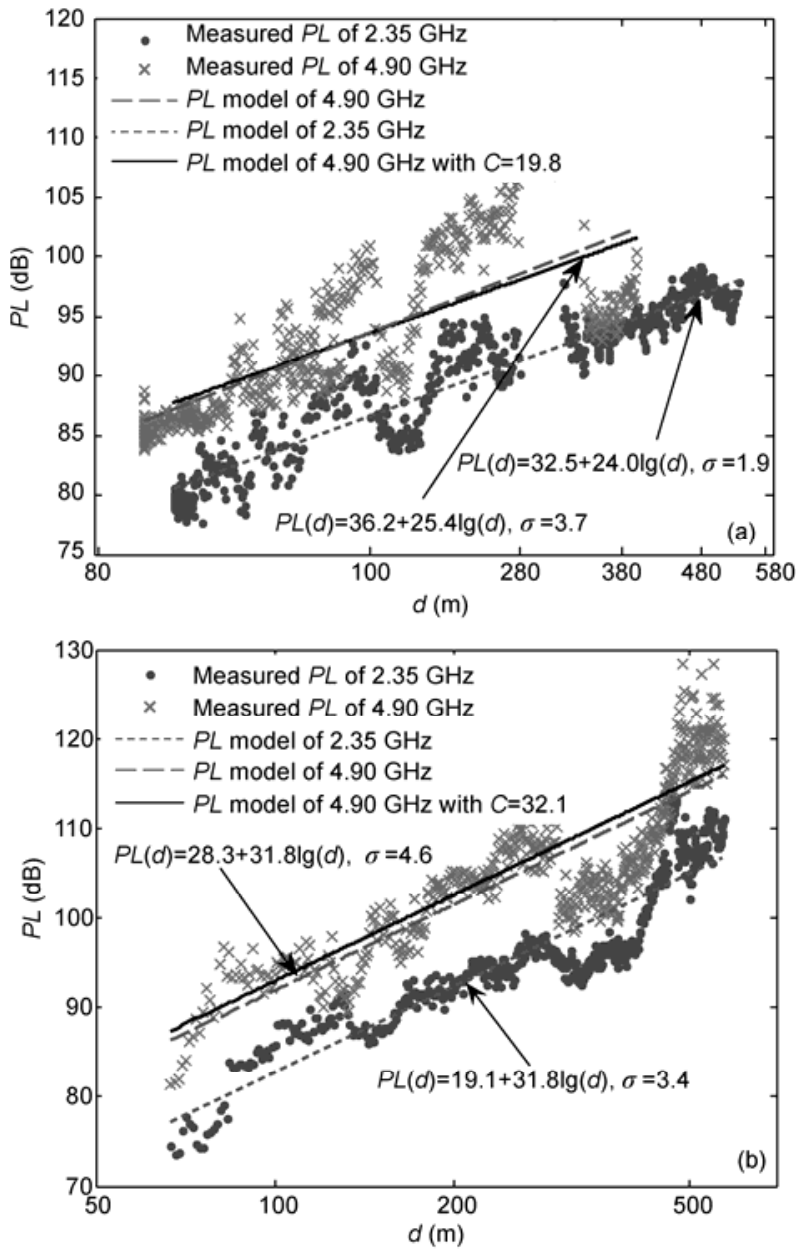

Figure 6 Frequency related factor models in a macrocell scenario. (a) Frequency dependent factor models in the LoS for a macrocell; (b) frequency dependent factor model in the NLoS for a macrocell.

of wireless communication, especially for orthogonal frequency division multiplexing (OFDM) systems. The delay spread (DS) or maximum excess delay determines the length of the cyclic prefix (CP). Normally, The length of CP is $2-4$ times the delay spread. As empirical data demonstrate, 3 times the delay spread can include $90 \%$ of the multipath bins. The maximum excess delay depends on the power threshold of the power delay profile (PDP) division. So in general the threshold should be given for counting identifiable delay bins. Furthermore, the coherence bandwidth, corresponding to the delay spread, is of great importance in deciding the subcarrier spacing and frequency domain pilot density in OFDM systems.

\subsection{Delay domain statistic model}

In a wideband mobile communication system, the transmitted signal will be dispersed in the time domain due to multipath propagation. The delay of the measured CIRs is the absolute transmitted delay, i.e., the delay $\tau_{l}$ introduced by the distance between TX and RX is removed. The parame- 
ters describing the delay are mainly the mean excess delay $\bar{\tau}$, root-mean-square (RMS) delay spread $\sigma_{\tau}$, and maximum excess delay $\tau_{\max }$. In the PDP, we assume that the $\ell$ th path power is $p_{\ell}=P_{a v}\left(\tau_{\ell}\right)$. The above definitions are mathematically written as follows.

(1) Mean excess delay $\bar{\tau}$ is the first moment of the PDP. It reflects the average arrival time of the main strong paths, compared to the first path.

$$
\bar{\tau}=\frac{\sum_{\ell=1}^{L} p_{\ell} \cdot \tau_{\ell}}{\sum_{\ell=1}^{L} p_{\ell}} .
$$

(2) RMS delay spread $\tau_{\text {rms }}$ is the second moment of the PDP. It reflects the degree of multipath diffusion in the time domain.

$$
\begin{aligned}
\tau_{\mathrm{rms}} & =\sqrt{\frac{\sum_{\ell=1}^{L} \tau_{\ell}^{2} \cdot p_{\ell}}{\sum_{\ell=1}^{L} p_{\ell}}-\left(\frac{\sum_{\ell=1}^{L} \tau_{\ell} \cdot p_{\ell}}{\sum_{l=1}^{L} p_{\ell}}\right)^{2}} \\
& =\sqrt{\frac{\sum_{\ell=1}^{L}\left(\tau_{\ell}-\bar{\tau}\right)^{2} \cdot p_{\ell}}{\sum_{l=1}^{L} p_{\ell}}} .
\end{aligned}
$$

(3) Maximum excess delay is the time-lag between the last and first signals to arrive at the PDP above a certain threshold, which reflects the maximum lag between the multipath signals arriving at the RX.

To acquire the statistical properties of delay spread, we provide the cumulative distribution function (CDF) of $\tau_{\mathrm{rms}}$ in Figure 7, based on numerous measurements in the SJZ macrocell at $580 \mathrm{MHz}, 2.35 \mathrm{GHz}$, and $4.9 \mathrm{GHz}$. The measurement setup is the same as in Section 2. The average of $\tau_{\text {rms }}$ varies from 110 to $40 \mathrm{~ns}$ in LoS as the frequency varies

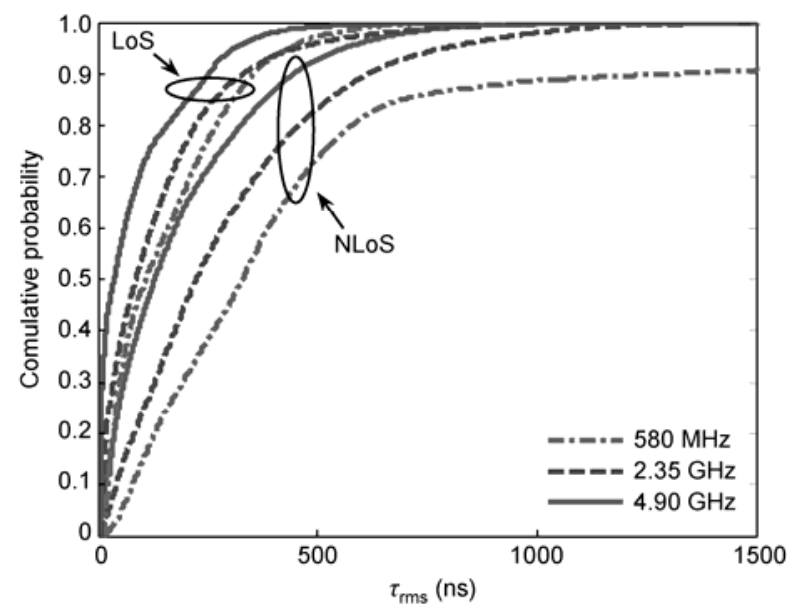

Figure 7 The empirical CDFs of RMS DS in an urban macrocell. from $580 \mathrm{MHz}$ to $4.9 \mathrm{GHz}$, while the average of $\tau_{\text {rms }}$ varies from 330 to $130 \mathrm{~ns}$ in NLoS. At the $10 \mathrm{MHz}$ bandwidth from 430 to $5750 \mathrm{MHz}$, the delay spread varies from 700 to $270 \mathrm{~ns}$, presenting the same trend. A rational explanation is that the radio signal can be absorbed easily at the higher carrier frequency with a shorter wavelength. When experiencing a reflection or transmitting a unit distance, the signal will exhibit greater loss. While path bins with bigger delays will experience more reflections and longer transmitted distances, these will lead to lower received power (even lower than the noise power) with lower frequency. It cannot be treated as a resolvable multipath component, so the delay spread is small.

\subsection{Spatial domain statistic model}

For wideband MIMO systems, besides the time and frequency characteristics, spatial characteristics are also very important and need to be further investigated. First, an accurate channel parameter estimation algorithm is required to extract the channel information contained in the measured CIR. With the estimation algorithm, the parameters of delay domain, spatial domain and Doppler domain can be acquired.

Three types of estimation algorithms are widely used for the extraction of wideband MIMO measurement data. The first category is the conventional beam-forming algorithms, which include the Bartlett beam-forming algorithm and the Capon minimum variance algorithm. Subspace based algorithms belong to the second type, which apply eigenvalue decomposition (EVD) or singular value decomposition (SVD) to acquire the signal subspace and noise subspace, and then use the characteristics of the subspace to estimate the direction of the received signal. Thus this type of algorithm is also called the eigen-structure based method [37,38]. Recently, the expectation maximization (EM) algorithm which is derived from the maximum likelihood estimation (MLE) has been proposed for the estimation of channel parameters [39]. The calculation of the maximum likelihood estimation of the parameters, even if the prior information is unknown and the observation data is imperfect, is a simple iteration algorithm. Thus its major advantages are simplicity and stability. Although the EM algorithm has the advantages of convenience and stability, it also has the disadvantage of slow convergence speed when there is a large amount of data.

Thus as an extension of the EM algorithm, the spacealternating generalized expectation maximization (SAGE) algorithm emerges with its fast convergent rate [40-44]. Due to the advantages of higher accuracy, availability for the estimation of parameters and applicability for almost every type of antenna array, the SAGE has become one of the most generally used channel estimation algorithms.

As illustrated in Figure 8, the SAGE algorithm divides the received signal $Y(t)$ into $L$ paths, i.e., $Y(t)=\sum_{l=1}^{L} X_{l}(t)$, and 


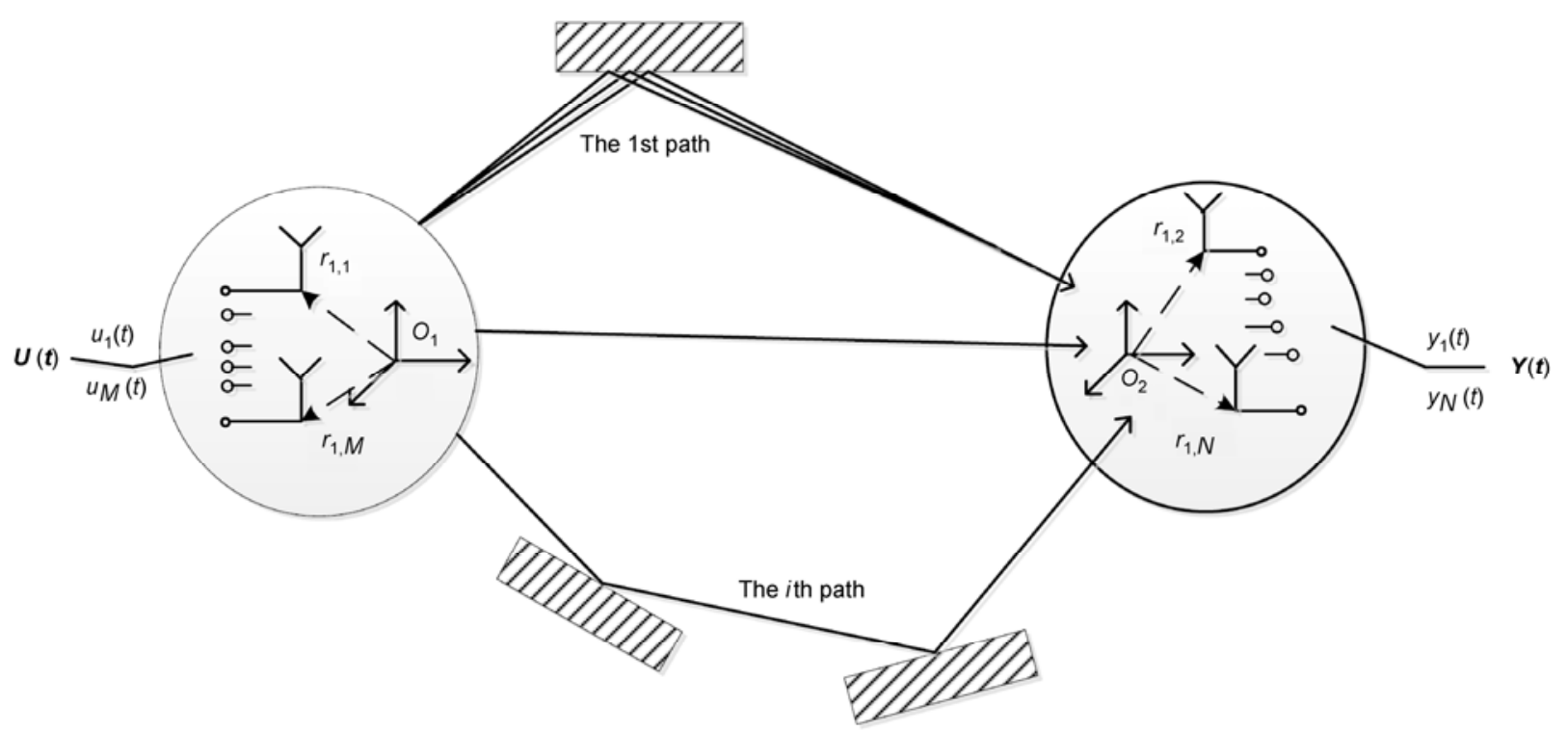

Figure 8 SAGE signal model.

$$
X_{l}(t) \triangleq s\left(t ; \boldsymbol{\theta}_{l}\right)+\sqrt{\frac{N_{0}}{2}} W_{l}(t), \quad l=1, \cdots, L .
$$

Assume that $X_{l}(t)=x_{l}(t), W_{l}(\mathrm{t})$ is the white noise. Then the likelihood function of $\theta_{l}$ is

$$
\begin{aligned}
\Lambda\left(\boldsymbol{\theta}_{l} ; x_{l}(t)\right) \propto 2 \Re & \{\underbrace{\left\{\int_{D_{0}} s^{*}\left(t ; \boldsymbol{\theta}_{l}\right) x_{l}(t) \mathrm{d} t\right\}}_{G_{1}} \\
& -\underbrace{\int_{D_{0}}\left|s\left(t ; \boldsymbol{\theta}_{l}\right)\right|^{2} \mathrm{~d} t}_{G_{2}} \propto z\left(\overline{\boldsymbol{\theta}}_{l} ; x_{l}\right),
\end{aligned}
$$

where $\Lambda(\cdot)$ is the likelihood function, $\theta_{l}$ is parmeter stacking the spatial and temporal parameters of the $l$ th path, $s\left(t ; \boldsymbol{\theta}_{l}\right)$ is the source signal and $x_{l}(t)$ is the corresponding signal observation.

When we calculate the estimation parameters in terms of maximizing the cost function, the SAGE algorithm calculates one parameter for one path at a time while keeping the other parameters constant, and the estimation of this parameter applies the newest updated values of the other parameters. Thus if there are $n$ parameters to be estimated, the SAGE algorithm turns the $n L$ dimensional estimation into 1 dimensional estimation times $n L$, which will reduce the complexity.

Figure 9 illustrates an example of AoD and AoA, which were estimated from the measurement data in the BJ urban macrocell (UMa) scenario. The asterisk represents the propagation paths with different powers and angles. The results show that even in the LoS propagation case the abundant scatterers will result in a large angular spread (AS).

In a MIMO communication system, the statistical models of the spatial parameters for both the transmitter and
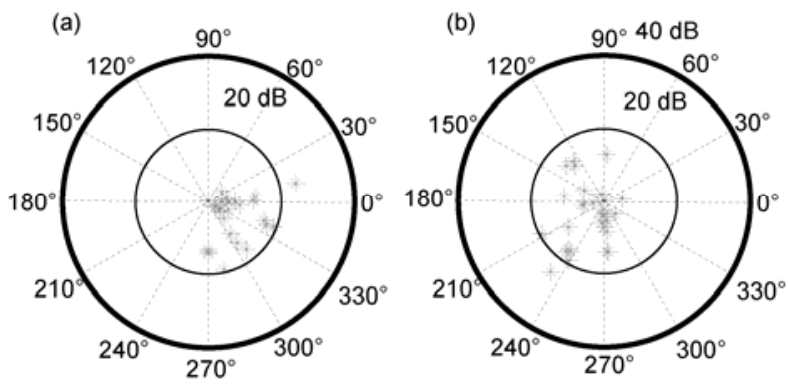

Figure 9 Example of the AoD (a) and AoA (b) for the LoS case in BJ.

receiver sides are very important. The parameters include the two dimensional AoA and AoD, AS, power angular spectrum (PAS) and coherent distance, etc. The two dimensional angular information stands for the horizontal angle and elevation angle, which are the key parameters of the wideband MIMO channel model. The two dimensional AoA and AoD information extracted from the measured data can be used to acquire the AS and PAS.

The AS of the channel, which is the second moment of the propagation angles, can be calculated using the method used for the delay spread. To avoid the problem of angular blur, a new method in the 3GPP spatial channel model (SCM) [45] is adopted for the calculation of the circular angular spread (CAS). Taking the AoD for example, $\phi_{\ell}$ is the AoD of the $\ell_{t h}$ path and $\varphi_{\ell}(\Delta)=\phi_{\ell}$. The AS is then defined as the minimum value for all the offset angles $\Delta$ :

$$
\begin{aligned}
\sigma_{r m s} & =\min _{\Delta} \sigma_{r m s}(\Delta) \\
& =\sqrt{\frac{\sum_{\ell=1}^{L}\left(\varphi_{\ell}(\Delta)-\mu(\Delta)\right)^{2} p_{\ell}}{\sum_{\ell=1}^{L} p_{\ell}}},
\end{aligned}
$$


and $\mu(\Delta)$ is

$$
\mu(\Delta)=\frac{\sum_{\ell=1}^{L} \varphi_{\ell}(\Delta) p_{\ell}}{\sum_{\ell=1}^{L} p_{\ell}},
$$

where $\varphi_{\ell}(\Delta)$ and $\varphi_{\ell}(\Delta)-\mu(\Delta)$ are normalized into the range of $[-\pi, \pi]$, i.e.,

$$
\varphi= \begin{cases}2 \pi+\varphi & \text { if } \varphi<-\pi, \\ \varphi & \text { if }|\varphi| \leqslant \pi, \\ 2 \pi-\varphi & \text { if } \varphi>\pi .\end{cases}
$$

The characteristics of the PAS of the AoA and AoD can be analyzed with the measured data. According to the specific number and distribution of scatterers in the surrounding environment, the PAS typically follows a uniform distribution, Gaussian distribution, Laplacian distribution or von-Mises distribution. We can acquire the spatial correlation of the MIMO antennas with the measured PAS or CIR.

In Table 2, the statistical values of CAS for the urban microcell scenario in SJZ and the urban macrocell scenario in BJ are depicted. In the table, $\mu$ and $\sigma$ are the parameters of the log-normal distributions when they are fitted to the CAS observations. The results in both scenarios show that for the same side, the CAS for the NLoS case is nearly the same as for the LoS case. Compared with urban macro-BJ at $2.35 \mathrm{GHz}$, the CASs of the AoA and AoD of a micro-SJZ at $2.35 \mathrm{GHz}$ are slightly smaller. In an urban macrocell, BS covers a larger area than that in the microcell. So the multipath components possibly arrive at the $\mathrm{Rx}$ via reflection from remote scatterers, i.e., high buildings, etc. Thus it leads to a larger AS at an urban macrocell. Such propagation environment characteristic results in our CASs for both AoA and AoD being larger than those of WINNER, i.e., the dense buildings and the obviously higher buildings, etc. The results given above indicate that we could explore the MIMO technique for the IMT-Advanced system for the larger AS, which would lead to the high system capacity [46].

Table 2 CAS statistics for the AoA and AoD

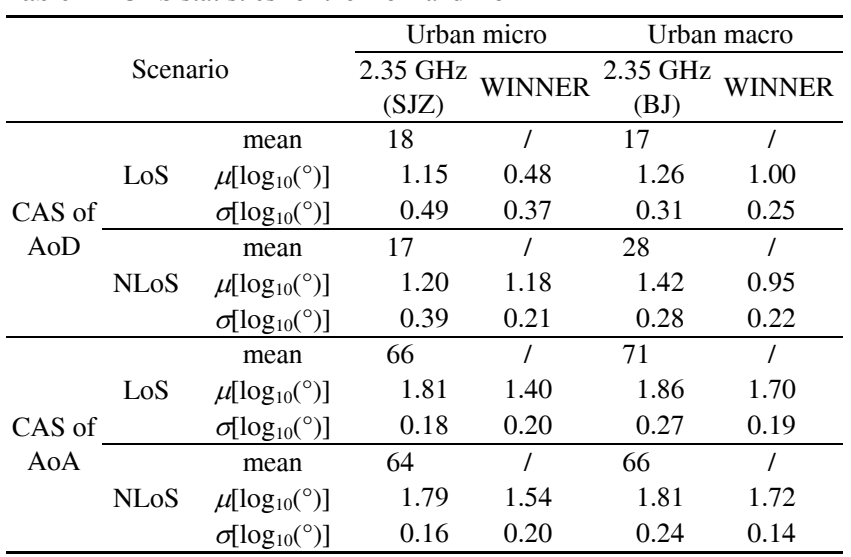

\section{MIMO channel models}

Channel modeling involves researching how to construct the mathematical model based on the theoretical analysis or empirical data, which accurately simulates the real propagation environment. We can classify the current modeling methodology into two types, i.e., one is the physical model and the other is the analytical channel model as shown in Figure 10.

Physical channel models mainly calculate the MIMO channel matrix with specific parameters and their distributions, including the delay, angle, complex gain of the multipath, antenna pattern and so on; whereas the analytical channel model reconstructs the MIMO channel matrix using its Eigen structure. Thus we can consider the analytical channel model as a re-abstract on the basis of the physical channel model from the perspective that it emphasizes the relationship between the MIMO channel coefficient matrix with CIR, while ignoring the specific propagation process.

\subsection{Physical channel models}

Physical channel models can also be categorized into two types: the GBSM and non-geometry stochastic channel model. For GBSM, the delays, angles and complex gains of the multipath, which relate to the propagation geometry, are generated according to the corresponding statistical distributions. The COST 259 directional channel model (DCM) [47], COST 273 channel model [48], 3GPP SCM [45,49], SCM extended (SCME) [50], IEEE 802.11n channel model [51] and the WINNER channel model [52] all belong to GBSMs. For the non-geometry based stochastic models, the random parameters are generated according to the probability distribution functions without regarding any propagation geometry information. The Wallace and Jensen channel models [53,54], which extended the Saleh-Valenzuela model [55], and Zwick model [56] are typical non-geometry based stochastic models.

\subsection{Analytical channel model}

Analytical channel models directly describe the characteristics of the MIMO channel matrix, and thus can be applied in the design, analysis and evaluation of the MIMO algorithms. Analytical channel models can also be categorized into two types, i.e., correlation based and propagation based analytical models.

The correlation based channel model reconstructs the MIMO matrix according to the correlation coefficients of each sub channel. The conventional correlation based channel model is the Kronecker model [57], which assumes separable correlation matrices for the transmitting and receiving sides. The Weichselberger channel model breaks through the limitations of a separable PAS for the Kronecker model, and constructs the model on the basis of the 


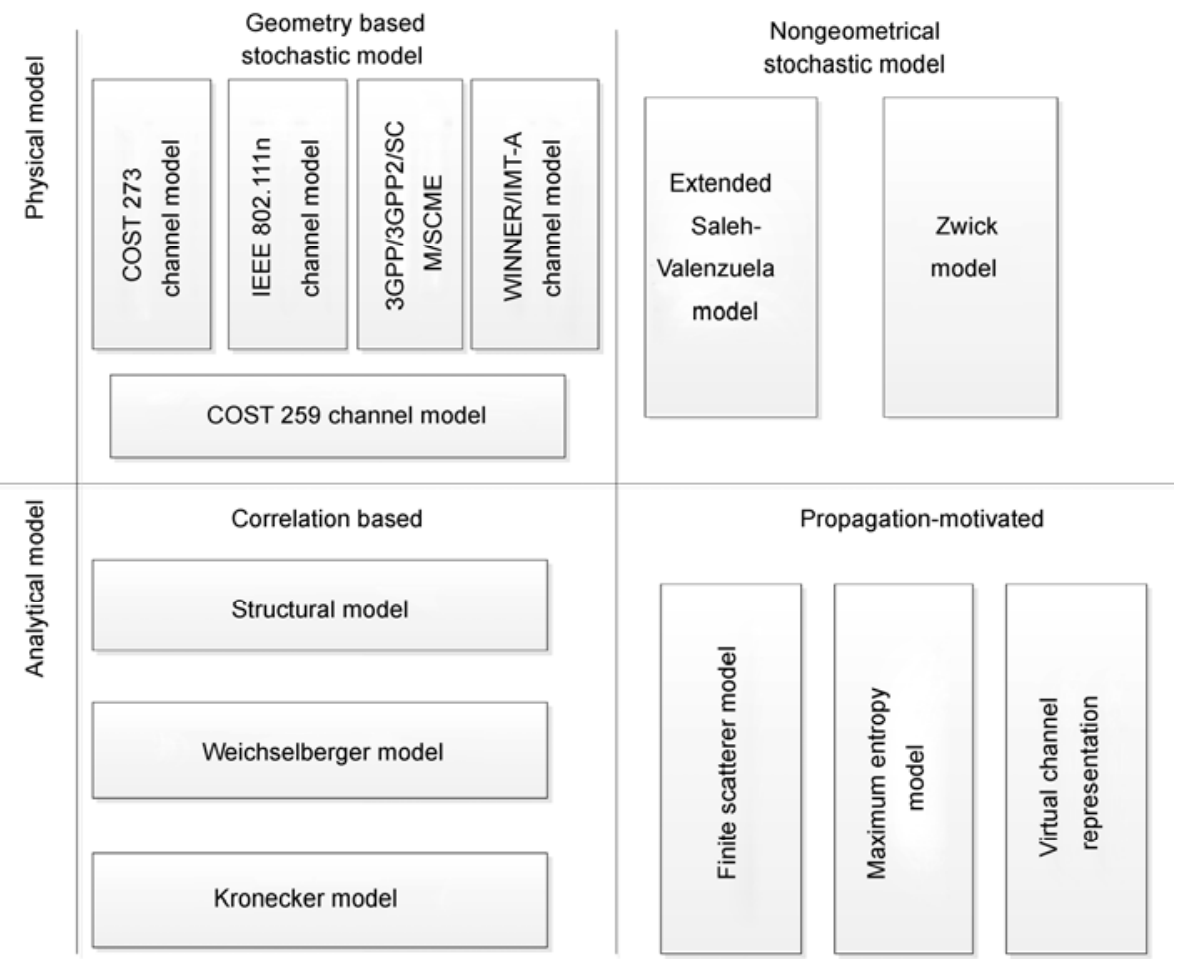

Figure 10 MIMO channel model classification.

eigenvalue decomposition of the correlation matrix [58]. The structural model extended from Weichselberger by Costa and Haykin can also be categorized into this type of analytical model. The i.i.d Gaussian channel model, which is widely used in the research of MIMO technology, is also a special case of the correlation model, and its correlation matrix is an identity matrix.

The propagation-motivated channel model and other physical models use the propagation environment and system parameters to reconstruct the channel. The difference is that the propagation-motivated channel model directly generates the probability distribution of the MIMO channel matrix with the characteristic parameters; whereas the other physical models first generate the parameters of the multipath, and then calculate the coefficients of the channel matrix elements. The finite scatterer model [59], maximum entropy model [60] and virtual channel representation [61] are all propagation-motivated channel models.

\subsection{Channel model simplification}

The widely used COST 207 channel model for GSM and the ITU-R M.1225 channel model for IMT-2000 are both tapped delay line (TDL) channel models. Since the system bandwidths are relatively narrow, the maximum tap number is 6. Moreover, these two channel models only consider single input single output (SISO) cases.

The IMT-Advanced channel model is a geometry-based stochastic channel model, which adopts the multipath superposition method to generate channel coefficients. In the
IMT-Advanced system, the bandwidth is up to $100 \mathrm{MHz}$ and the MIMO antennas are utilized for both the transmitter and receiver. Thus it is necessary to model not only the delay and Doppler domain; but also the spatial domain and the correlation of the parameters. The number of random parameters for the IMT-Advanced channel model is greatly increased compared with the GSM and IMT-2000 channel models. Moreover, compared with the COST 207 and M.1225 channel models, the PDP is generated according to the probability distributions rather than to constants. All these differences result in a much higher computation complexity for IMT-Advanced channel model and these require a lot of simulation work considering there are only 4 mandatory environments, each with both LoS and NLoS cases.

Based on the analysis of measured data and discussion with the ITU-R channel model drafting group, we propose five simplification methods to reduce the simulation complexity while retaining flexibility for configuring the antenna arrays and geometries as for the GBSM. The five simplification methods are:

SM-A: clip clusters with power $<P_{t h}$.

SM-B: Fix DS at its mean value.

SM-C: Fix cross-polarization discrimination (XPD) at its mean value.

SM-D: remove cross-correlations between large scale parameters.

SM-E: SM-A, B, C and D.

Here, we call the IMT-Advanced channel model the baseline model. The baseline model divides the whole scenario into channel segments, and in each channel segment 
the channel power distribution is described by the DS, angular spread of departure (ASD), angular spread of arrival (ASA), standard deviation of shadow fading and Rician $\mathrm{K}$-factor. These five parameters are called the large scale parameters (LSP) of the channel model. We can denote them by $\tau_{r m s}, \phi_{r m s}, \varphi_{r m s}, \sigma_{\xi}$ and $K$, which follow the five dimensional log-normal distribution as:

$$
\begin{aligned}
L & =\left(\log _{10} \tau_{r m s}, \log _{10} \phi_{r m s}, \log _{10} \varphi_{r m s}, 10 \log _{10} \sigma_{\xi}, 10 \log _{10} K\right)^{\mathrm{T}} \\
& \sim \mathcal{N}_{5}\left(\mu_{L}, \sigma_{L}\right) .
\end{aligned}
$$

LSPs are regenerated for different channel segments, whereas in the same channel segment only the multipath fading changes while the LSPs remain the same.

According to Kyösti's analysis, the computational complexity of channel model simulation is divided into three different categories: (1) the complexity of channel coefficient generation, (2) the number of required parameters, and (3) the complexity of simulation. Both (1) and (3) are proportional to the number of delay taps. Hence, the computational complexity can be reduced if the number of delay taps can be reduced. Thus we propose to clip some taps considering the fact that the average power of some clusters is relatively low with respect to the maximum cluster power, e.g., $20 \mathrm{~dB}$ lower. However, the impact of reducing the number of delay taps needs to be investigated to retain the accuracy of the models.

In the IMT-Advanced channel model, a cluster is defined as a propagation path diffused in space, in either or both delay and angle domains. Consider a scenario with $N$ clusters, where the average cluster power of the $n_{t h}$ cluster is $P_{n}$ in decibels. Denote the cluster indexing set as $\mathcal{I}=\{1,2, \cdots$, $N\}$. For a given cluster power threshold, $P_{t h}$ in decibels, the cluster is clipped if its power is below this threshold when the power of the dominant cluster is chosen as a reference. The reduced number of clusters is a random variable, i.e.,

$$
N_{\text {clipped }}=\sum_{n=1}^{N} I\left(P_{n}+P_{t h}<\max _{k \in \mathcal{I}} P_{k}\right),
$$

where $I(A)$ is the indicator function of event $\mathcal{A}$, namely:

$$
I(\mathcal{A})=\left\{\begin{array}{ll}
1, & \text { event } \mathcal{A} \text { is true } \\
0, & \text { otherwise }
\end{array} .\right.
$$

The computational time for simulation is dominated by the convolution operation, and the time required by such an operation is proportional to the number of delay taps (or the number of clusters). Consequently, if we normalize the computational time before and after clipping, the normalized computational time (NCT) can be defined as the ratio of the average number of remaining clusters to the number of original clusters, that is,

$$
T_{\text {norm }}\left(P_{t h}\right)=1-\frac{1}{N} E\left(N_{\text {clipped }}\right) .
$$

Figure 11(a) shows the relationship between the NCT and the clipping threshold. It shows that the average computational complexity can be reduced by more than $40 \%$ when a $25 \mathrm{~dB}$ clipping threshold is adopted for the "Indoor LoS" case, while a $15 \%$ improvement can be expected for the "Outdoor NLoS" case if $P_{t h}=15 \mathrm{~dB}$. The NLoS case requires a higher clipping threshold with respect to the LoS case to achieve the same NCT reduction. As for the LoS case, the power of an LoS ray is stronger than the NLoS rays such that most clusters were clipped out for a low threshold. For the NLoS case, the power difference between clusters is not as large as for the LoS case. Therefore, even with a lower threshold, clusters are more likely to be clipped. To keep the total power of the remaining clusters unitary, the loss of the power of the clipped clusters needs to be compensated for in the remaining clusters.

A direct consequence of clipping clusters is the bias in the RMS DS which is inversely proportional to the coherent bandwidth, a critical parameter for wireless systems. For a given drop, we denote the RMS DS before and after clipping
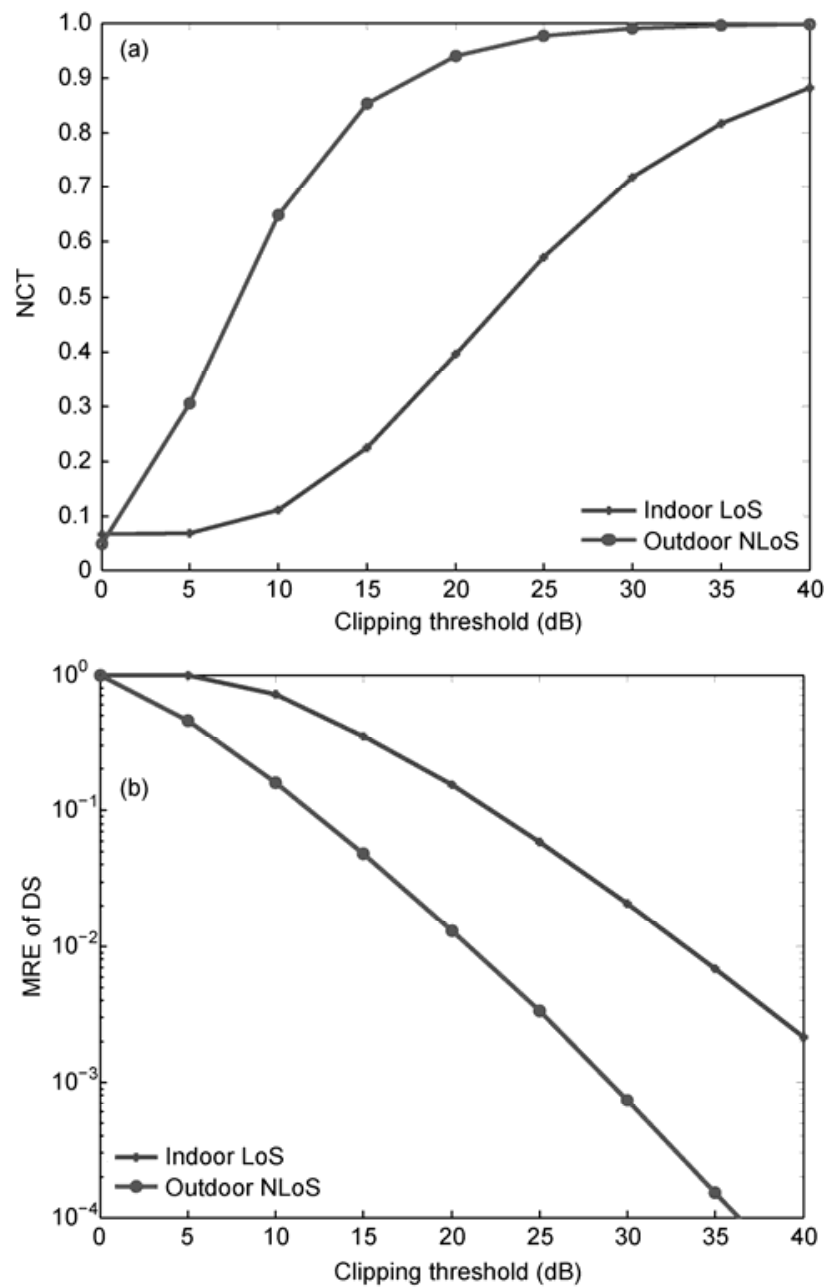

Figure 11 The impact of the clipping threshold on the efficiency and accuracy (averaged over $10^{4}$ drop runs). (a) NCT versus clipping threshold; (b) MRE of DS versus clipping threshold. 
with a threshold $P_{t h}$ as $\tau_{\text {rms }}$ and $\tilde{\tau}_{\text {rms }}$, respectively. The mean relative error (MRE) of the RMS DS versus the clipping threshold is defined by:

$$
\varepsilon_{D S}\left(P_{t h}\right)=E\left(\frac{\left|\tilde{\tau}_{\mathrm{rms}}-\tau_{\mathrm{rms}}\right|}{\tau_{\mathrm{rms}}}\right) .
$$

The MRE of the RMS DS is plotted in Figure 11(b). It shows, as expected, that as the threshold becomes larger, the relative error $\varepsilon_{D S}$ becomes smaller. The MRE of DS is more sensitive to the clipping threshold in the NLoS case. In particular, $\varepsilon_{D S}$ is around 5\% when the clipping threshold $P_{t h}=15 \mathrm{~dB}$ for the outdoor NLoS case or $P_{t h}=25 \mathrm{~dB}$ for the indoor LoS case.

Thus we can use the clipping threshold of 25 or $15 \mathrm{~dB}$ for indoor LoS and NLoS conditions respectively to reduce the complexity of the simulation with an acceptable loss in performance. This clipping cluster scheme was adopted by the ITU-R in the second session of the conference of WP5D in June 2008. At the same time, we also proposed to fix the RMS DS and XPD to their mean values, and remove the correlation between the LSPs to further simplify the channel model. All the simplifications are based on the structure of GBSM, and thus the antenna arrays and link geometry can be flexibly configured.

\section{Open topics}

Numerous studies, both national and international, have measured and modelled the wideband MIMO channel. Our achievements have been included in the standardization of the ITU-R IMT-Advanced channel model. This research is fundamental for the evaluation and optimization of an IMT-Advanced system. However, with the development of new technologies, the new challenges and requirements in the research on channels emerge as follows.

(1) Propagation characteristics under relay systems should be well understood. In the World Radio-communication Conference 2007 (WRC-07), the frequency band of $3.2-3.4 \mathrm{GHz}$ is allocated to the IMT-Advanced system. The higher carrier frequency will lead to a smaller coverage area, and thus frequent handovers exist. The users that locate at the cell edges can barely acquire a reasonable data rate and quality of service (QoS). The relay is known to be a very promising technology to resolve these problems. There are various ways to categorize the relay. Traditionally relay is classified into amplify-and-forward relay and decodeand-forward relay according to the different signal processing schemes at the relay node. According to the mobility of the relay, it can be categorized into either a fixed or mobile relay. However, due to the difficulties in measuring the multi-link structure of the relay system, most of the relay channel models are based on hypotheses rather than measurements. Although IEEE 802.16 has proposed several evaluation scenarios and models for a fixed relay, the models lack support from measurement data and thus have not been accepted by other standardization organizations. Much measurement and modeling work needs to be done in the research on relay systems, including mobile relays which are likely to be used for communicating with mobiles in high-speed trains or cars. There are some preliminary reports [62,63], but more thorough work is required.

(2) Channel measurement and modeling for the higher frequency band are seldom carried out. The frequency resource is limited and valuable. With the development of wireless communication, the low frequency bands have already been allocated to existing services. Thus for future communication system, high frequency bands will be used. The measurement work has already been started, and the carrier frequencies include 5-15 GHz and even $60 \mathrm{GHz}$. Since 2009 the National High-tech R\&D Program of China (863 Program) has already been carrying out propagation characteristics research at 6-15 GHz. However, much work still remains to be done for the higher frequency bands.

(3) Channel measurement and modeling for femtocell application are necessary. With the development of $3 \mathrm{G}$ communication systems and the trend in mobile broadband, femtocells that use mini base stations have emerged for the needs of short distance communication. For indoor users, it is possible to install a low-power device to construct the femtocell to further enhance the coverage and throughput. The diameter of the coverage area of a femtocell is quite small, typically $20-50 \mathrm{~m}$, and the transmit power is very low. In such circumstances, the hypotheses of plane wave propagation and antenna far field are not reasonable for such short distances. Thus the existing channel models may no longer be applicable for femtocell use. Dedicated channel research is required for the development of the femtocell.

(4) Multilink channel measurement and modeling should be investigated. For system level simulation of multilink systems, the channel coefficients of multiple links are required at the same time. Thus the correlations of the large and small scale parameters between different links need to be precisely modeled. Although the SCM, SCME and WINNER II channel models include the correlation of large scale parameters, modeling methods and results are not uniform, or even not compatible. Moreover, multilink simulation in different scenarios is not supported by existed channel models. More thorough work still exists for channel researchers.

This work was supported by the National Natural Science Foundation of China (61171105 and 61121001), National Key Technology Research and Development Program of the Ministry of Science and Technology of China (2012BAF14B01), National Science and Technology Major Project of the Ministry of Science and Technology of China (2009ZX03007-003-01). The authors express their gratitude to all the colleagues and students who helped to finish the measurements regardless of the cold winter or hot 
summer, especially to Dr. Zhang Ming, Dr. Gao Xinying, Dr. Zhang Yu, Dr. Nie Xin, Mr. Sheng Nan, Mr. Xu Ding, Mr. Lu Yang, Miss Liang Yanping, Miss Huang Chen, Mr. Dong Di, Mr. Hu Shiwei, Mr. Wu Zhiwen, Mr. Huang Chengxiang, Mr. Tian Lei and Mr. Zhang Fenghua. Thanks to Dr. Gao Yu from Elektrobit for arranging to export the equipment to China in 2005, thus making this research possible. Finally, thanks to Dr. Wu Yiyan from Motorola, Dr. Liu Guangyi and Mr. Dong Weihui from the China Mobile Research Institute and Dr. Prof. Mansoor Shafi from New Zealand Telecommunications for their continuous support of this research.

1 Bi Q, Zysman G L, Menkes H. Wireless mobile communications at the start of the 21th century. IEEE Commun Mag, 2001, 39: 110-116

2 Tian J F, Zheng X Y, Hu H L, et al. A survey of next generation mobile communications research in China. Chin Sci Bull, 2011, 56: 2875-2888

3 ITU-R M.1645-E. Framework and overall objectives of the future development of IMT-2000 and systems beyond IMT-2000, 2003

4 Molisch A F. Wireless communications. Chichester, UK: John Wiley \& Sons Ltd., 2005

5 Winters $\mathbf{J} \mathrm{H}$. On the capacity of radio communication systems with diversity in a Rayleigh fading environment. IEEE J Sel Area Commun, 1987, 5: 871-878

6 Foschini G J, Gans M J. On limits of wireless communications in a fading environment when using multiple antennas. Wireless Personal Commun, 1998, 6: 311-335

7 Telatar I E. Capacity of multi-antenna Gaussian channels. European Trans Telecommun, 1999, 10: 585-595

8 Rice S O. Mathematical analysis of random noise. Bell Sys Technical J, 1944, 23: 282-332

9 Failli M. Digital land mobile radio communications-COST 207. Luxemberg: European Union, 1989

10 ITU-R M.1225. Guidelines for evaluation of radio transmission technologies for IMT-2000, 1997

11 Sampath H, Talwar S, Tellado J, et al. A fourth-generation MIMOOFDM broadband wireless system: Design, performance, and field trial results. IEEE Commun Mag, 2002, 40: 143-149

12 Dubuc C, Starks D, Creasy T, et al. A MIMO-OFDM prototype for next-generation wireless WANs. IEEE Commun Mag, 2004, 42: $82-87$

13 IST-4-027756 WINNER II D1.1.2 v1.2 WINNER II channel models, Feb. 2008. [Online]. Available: http://www.ist-winner.org/WINNER2Deliverables/D1.1.2.zip

14 Ohta Y, Fujii T. Delay profile prediction in microwave-band for wideband radio propagation. In: Proceedings of International Symposium on Antennas and Propagation, 2005, Seoul, Korea. Washington DC: IEEE, 2005. 1105-1108

$15 \mathrm{Kim} \mathrm{J} \mathrm{H}$. The wideband characteristics of radio propagation at $8 \mathrm{GHz}$ band. In: Proceedings of Asia-Pacific Conference on Communications, 2007 Oct., Bangkok, Thailand. Washington DC: IEEE, 2007. 91-94

16 Zhang J H, Dong D, Liang Y P, et al. Propagation characteristics of wideband MIMO channel in urban micro- and macro-cell. In: Proceedings of the 19th International Symposium on Personal, Indoor and Mobile Radio Communications, 2008 Sept. 15-18, Poznan Poland. Washington DC: IEEE, 2008. 1-6

17 Zhang J H, Gao X Y, Zhang P, et al. Propagation characteristics of wideband MIMO channel in hotspot area at 5.25 GHz. In: Proceedings of the 18th International Symposium on Personal, Indoor and Mobile Radio Communications, 2007 Sept. 3-7, Athens, Greece. Washington DC: IEEE, 2007. 1-6

18 Zhang Y, Zhang J H, Peter J S, et al. Reduced complexity channel models for IMT-Advanced evaluation. EURASIP J Wireless Commun Networking, 2009, doi: 10.1155/2009/195480

19 Gao X Y, Zhang J H, Liu G Y, et al. Large-scale characteristics of $5.25 \mathrm{GHz}$ based on wideband MIMO channel measurements. IEEE Ant Wireless Propag Lett, 2007, 6: 263-266

20 ITU-R 8F/1252-E. Proposed new test environments and channel models of preliminary draft new report IMT.EVAL. In: 22nd Meet- ing of Working Party 8F, 2007 May 23-31, Kyoto

21 ITU-R 5D/205-E. Proposed layout update of indoor hotspot scenario for IMT-Advanced evaluation. In: 2nd Meeting of Working Party 5D, 2008 Jun. 24-Jul. 2, Dubai

22 ITU-R 5D/207-E. Proposed to simplify the propagation model for IMT-Advanced evaluation. In: 2nd Meeting of Working Party 5D, 2008 Jun. 24-Jul. 2, Dubai

23 Rappaport T S. Wireless communications: Principles and practice. Upper Saddle River, USA: Prentice Hall PTR. 2002

24 Cox D C. Delay Doppler characteristics of multipath delay spread and average excess delay for $910 \mathrm{MHz}$ urban mobile radio paths. IEEE Trans Ant Propag, 1972, 20: 625-635

25 Rappaport T S. Characterization of UHF multipath radio channels in factory buildings. IEEE Trans Ant Propag, 1989, 37: 1058-1069

26 Taparugssanagorn A, Ylitalo J. Empirical characteristics of urban macrocell multiple-input multiple-output channel at $2.53 \mathrm{GHz}$. In: Proceedings of the first European Conference on Antennas and Propagation, 2006 Nov. 6-10, Nice, France. Washington DC: IEEE, 2006. 1-5

27 Richter A, Hampicke D, Sommerkorn G, et al. MIMO measurement and joint M-D parameter estimation of mobile radio channels. In: Proceedings of the 53rd Vehicular Technology Conference, 2001 May. 6-9, Rhodes, Poland. Washington DC: IEEE, 2001. 214-218

28 Butt G, Parks M, Evans B G. Measurements and modelling of wireless channel for satellite mobile/personal communication systems. In: Proceedings of Tenth International Conference on Digital Satellite Communications, 1995 May. 15-19, Brighton, Britain. Washington DC: IEEE, 1995. 106-114

29 Baum D S, Gore D, Nabar R, et al. Measurement and characterization of broadband MIMO fixed wireless channels at $2.5 \mathrm{GHz}$. In: Proceedings of IEEE International Conference on Personal Wireless Communications, 2000 Dec. 17-20, Hyderabad, India. Washington DC: IEEE, 2000. 203-206

30 Zhang P, Tao X F, Zhang J H, et al. A vision from the future: Beyond 3G TDD. IEEE Commun Mag, 2005, 43: 38-44

31 Okumura Y, Ohmuri E, Kawano T, et al. Field strength and its variability in VHF and UHF land mobile services. Rev Elec Commun Lab, 1968, 16: 825-873

32 Hata M. Empirical formula for propagation loss in land mobile radio services. IEEE Trans Veh Technol, 1980, 29: 317-325

33 Walfisch J, Bertoni H L. A theoretical model of UHF propagation in urban environments. IEEE Trans Ant Propag, 1988, 36: 1788-1796

34 Ikegami F, Yoshida S, Takeuchi T, et al. Propagation factors controlling mean field strength on urban streets. IEEE Trans Ant Propag, 1984, 32: 822-829

35 Damosso E, Correia L M. Digital mobile radio towards future generation systems: COST231 final report. Brussels, Belgiunm: European Commission, 1999

36 Kivinen J, Zhao X, Vainikainen P I. Empirical characterization of wideband indoor radio channel at $5.3 \mathrm{GHz}$. IEEE Trans Ant Propag, 2001, 49: 1192-1203

37 Roy R, Paulraj A, Kailath T. ESPRIT - A subspace rotation approach to estimation of parameters of cisoids in noise. IEEE Trans Acoustic, Speech, Signal Proc, 1986, 34: 1340-1342

38 Roy R, Kailath T. ESPRIT - Estimation of signal parameters via rotational invariance techniques. IEEE Trans Acoustic, Speech, Signal Proc, 1989, 37: 984-995

39 Poor H V. An Introduction to Signal Detection and Estimation. New York: Springer-Verlag, 1988

40 Fessler J A, Hero A O. Space-alternating generalized expectation maximization algorithm. IEEE Trans Signal Proc, 1994, 42: 26642677

41 Fleury B H, Tschudin M, Heddergott R, et al. Channel parameter estimation in mobile radio environments using the SAGE algorithm. IEEE J Sel Area Commun, 1999, 17: 434-450

42 Fleury B H, Jourdan P, Stucki A. High-resolution channel parameter estimation for MIMO applications using the SAGE algorithm. In: Proceedings of International Zurich Seminar on Broadband Communications. Accessing, Transmission, Networking, 2002 Feb 15-17, 
Zurich, Switzerland. Washington DC: IEEE, 2000. 30: 1-9

43 Yin X, Fleury B H, Jourdan P, et al. Doppler frequency estimation for channel sounding using switched multiple transmit and receive antennae. In: Proceedings of Global Communication Conference, 2003 Dec. 1-5, San Francisco, USA. Washington DC: IEEE, 2003. 4: 2177-2181

44 Fleury B H, Yin X, Rohbrandt K G, et al. Performance of a highresolution scheme for joint estimation of delay and bidirection dispersion in the radio channel. In: Proceedings of the 55th IEEE Vehicular Technology Conference, 2002 May 6-9, Alabama, USA. Washington DC: IEEE, 2002. 522-526

45 3GPP TR 25.996. 3rd Generation partnership project technical specification group radio access networks, spatial channel model for MIMO simulations, June. 2003. [Online]. Available: http://www. 3gpp.org/ftp/Specs/archive/25_series/25.996/25996-100.zip

46 Bolcskei H, Gesbert D, Paulraj A J. On the capacity of OFDM- based spatial multiplexing systems. IEEE Trans Commun, 2002, 50: 225234

47 Correia L M. Wireless Flexible Personalized Communications: COST 259, European Co-operation in Mobile Radio Research. Chichester, UK: John Wiley \& Sons, Ltd, 2001

48 Correia L M. Mobile Broadband Multimedia Networks: Techniques, Models and Tools for 4G. Oxford, UK: Academic Press, 2006

49 Calcev G, Chizhik D, Göransson B, et al. A wideband spatial channel model for system-wide simulations. IEEE Tran Veh Technol, 2007, 56: $389-403$

50 Baum D S, Hansen J, Salo J. An interim channel model for beyond-3G systems: Extending the 3GPP spatial channel model (SCM). In: Proceedings of the 61st IEEE Vehicular Technology Conference, 2005 May 30-Jun. 1, Stockholm, Sweden. Washington DC: IEEE, 2005. 3132-3236

51 Erceg V, Schumacher L, Kyritsi P, et al. IEEE P802.11 wireless LANs: TGn channel model (IEEE 802.11-03/940r4). May. 2004. [Online]. Available: 11-09-0308-00-00ac-tgac-channel-model-addendum-document.doc
52 IST-2003-507581 WINNER D5.4 v1.4 Final report on link level and system level channel models, Nov. 2005. [Online]. Available: http://www.ist-winner.org/DeliverableDocuments/D5.4.pdf

53 Wallace J W, Jensen M A. Statistical characteristics of measured MIMO wireless channel data and comparison to conventional models. In: Proceedings of the 54th IEEE Vehicular Technology Conference, 2001 Oct 7-11, Sidney, Australia. Washington DC: IEEE, 2001. 2: 1078-1082

54 Wallace J W, Jensen M A. Modeling the indoor MIMO wireless channel. IEEE Trans Ant Propag, 2002, 50: 591-599

55 Saleh A, Valenzuela R. A statistical model for indoor multipath propagation. IEEE J Sel Area Commun, 1987, 5: 128-137

56 Zwick T, Fischer C, Wiesbeck W. A stochastic multipath channel model including path directions for indoor environments. IEEE J Sel Area Commun, 2002, 20: 1178-1192

57 Chuah C N, Kahn J M, Tse D. Capacity of multi-antenna array systems in indoor wireless environment. In: Proceedings of Global Communication Conference, 1998 Nov. 8-12, Sydney, Australia. Washington DC: IEEE, 1998. 1894-1899

58 Weichselberger W, Herdin M, Zcelik H O, et al. A stochastic MIMO channel model with joint correlation of both link ends. IEEE Trans Wireless Commun, 2006, 5: 90-99

59 Burr A. Capacity bounds and estimates for the finite scatterers MIMO wireless channel. IEEE J Sel Area Commun, 2003, 21: 812-818

60 Debbah M, Muller R. MIMO channel modeling and the principle of maximum entropy. IEEE Trans Inf Theory, 2005, 51: 1667-1690

61 Sayeed A M. Deconstructing multi-antenna fading channels. IEEE Trans Signal Proc, 2002, 50: 2563-2579

62 Zhang J H, Dong D, Ling Y P, et al. Propagation characteristics of wideband relay channels in urban environment. IEEE Ant Wireless Propag Lett, 2010, 6: 657-661

63 Nie X, Zhang J H, Liu Z M, et al. Experimental investigation of MIMO relay channels statistics and capacity based on wideband outdoor measurements at $2.35 \mathrm{GHz}$. Sci China Inf Sci, 2011, 54: 19451956

Open Access This article is distributed under the terms of the Creative Commons Attribution License which permits any use, distribution, and reproduction in any medium, provided the original author(s) and source are credited. 